ARTICLE

\title{
Quadratic Fermi node in a 3D strongly correlated semimetal
}

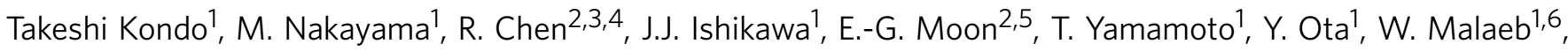

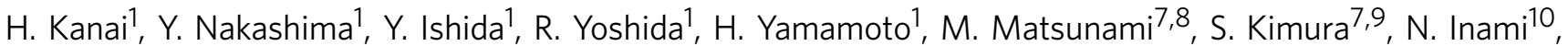 \\ K. Ono ${ }^{10}$, H. Kumigashira ${ }^{10}$ S. Nakatsuji,11, L. Balents ${ }^{12} \&$ S. Shin ${ }^{1}$
}

Strong spin-orbit coupling fosters exotic electronic states such as topological insulators and superconductors, but the combination of strong spin-orbit and strong electron-electron interactions is just beginning to be understood. Central to this emerging area are the $5 d$ transition metal iridium oxides. Here, in the pyrochlore iridate $\operatorname{Pr}_{2} \mid r_{2} \mathrm{O}_{7}$, we identify a non-trivial state with a single-point Fermi node protected by cubic and time-reversal symmetries, using a combination of angle-resolved photoemission spectroscopy and first-principles calculations. Owing to its quadratic dispersion, the unique coincidence of four degenerate states at the Fermi energy, and strong Coulomb interactions, non-Fermi liquid behaviour is predicted, for which we observe some evidence. Our discovery implies that $\mathrm{Pr}_{2} \mathrm{Ir}_{2} \mathrm{O}_{7}$ is a parent state that can be manipulated to produce other strongly correlated topological phases, such as topological Mott insulator, Weyl semimetal, and quantum spin and anomalous Hall states.

\footnotetext{
${ }^{1}$ ISSP, University of Tokyo, Kashiwa, Chiba 277-8581, Japan. ${ }^{2}$ Physics Department, University of California, Santa Barbara, California 93106, USA. ${ }^{3}$ Physics Department, University of California, Berkeley, California 94720, USA. ${ }^{4}$ Molecular Foundry, Lawrence Berkeley National Laboratory, Berkeley, California 94720, USA. ${ }^{5}$ Department of Physics, Korea Advanced Institute of Science and Technology, Daejeon 305-701, Korea. ${ }^{6}$ Physics Department, Faculty of Science, Beirut Arab University, P. O. Box 11-5020 Beirut, Lebanon. ${ }^{7}$ UVSOR Facility, Institute for Molecular Science, Okazaki 444-8585, Japan. ${ }^{8}$ Energy Materials Laboratory, Toyota Technological Institute, Nagoya 468-8511, Japan. ${ }^{9}$ Graduate School of Frontier Biosciences, Osaka University, Suita, Osaka 5650871, Japan. ${ }^{10}$ Institute of Materials Structure Science, High Energy Accelerator Research Organization (KEK), Tsukuba, Ibaraki 305-0801, Japan. ${ }^{11}$ PRESTO, Japan Science and Technology Agency (JST), 4-1-8 Honcho Kawaguchi, Saitama 332-0012, Japan. ${ }^{12}$ Kavli Institute for Theoretical Physics, Santa Barbara, California 93106, USA. Correspondence and requests for materials should be addressed to T.K. (email: kondo1215@issp.u-tokyo.ac.jp).
} 
F ollowing the discovery of topological insulators ${ }^{1-4}$, the next frontier is the regime in which both spin-orbit coupling and correlation effects are strong ${ }^{5-12}$. Theory has suggested that the pyrochlore iridates, a family of cubic $5 d$ transition metal oxides ${ }^{13,14}$, may realize both band inversion, the essential ingredient of topological insulators and strong correlations $\mathrm{s}^{9,12}$. Empirical evidence for the latter is plentiful. Notably, $\mathrm{Pr}_{2} \mathrm{Ir}_{2} \mathrm{O}_{7}$ appears to be proximate to an interactiondriven antiferromagnetic quantum critical point tuned by the A-site ionic radius, which is located between the two ions with largest radii, $\mathrm{A}=\mathrm{Pr}$ and $\mathrm{A}=\mathrm{Nd}^{14,15}$. It is the only compound among the series in which the iridium electrons remain paramagnetic and itinerant down to the lowest measured temperatures. It displays bad metallic behaviour and non-trivial spontaneous Hall transport, suggesting strong correlations ${ }^{6,15,16}$. Moreover, recent thermodynamic measurements have revealed zero-field quantum criticality without tuning ${ }^{17}$.

The phenomenological suggestion of Moon et al. ${ }^{11}$, whose implications are summarized in Fig. 1, is that the Fermi surface of $\mathrm{Pr}_{2} \mathrm{Ir}_{2} \mathrm{O}_{7}$ contains a single Fermi node at the $\Gamma$ point, which emerges as the touching point of two quadratically dispersing conduction and valence bands ${ }^{18}$. The presence of this touching is actually required by symmetry and group theory (the quadruplet at the zone centre lies in the $\Gamma_{8}$ representation of the double group of $\mathrm{O}_{\mathrm{h}}$ ), but its location directly at the Fermi energy was an ad hoc theoretical assumption. If the assumption is correct, $\mathrm{Pr}_{2} \mathrm{Ir}_{2} \mathrm{O}_{7}$ becomes a strongly correlated analogue of $\mathrm{HgTe}^{19,20}$, which has a mathematically identical quadratic node at the Fermi energy, and implies that $\mathrm{Pr}_{2} \mathrm{Ir}_{2} \mathrm{O}_{7}$ should be tunable into various topological states (see Fig. 1). Furthermore, theory has predicted that the quadratic nodal semimetal itself is fundamentally altered by long-range Coulomb interactions (negligible in $\mathrm{HgTe}$ due to the large dielectric constant, but not so here), becoming a non-Fermi liquid state. Thus the Fermi node, if correct, means that $\mathrm{Pr}_{2} \mathrm{Ir}_{2} \mathrm{O}_{7}$ is a natural parent material for strongly interacting topological phases and non-Fermi liquid states.

In this paper, we focus on the phenomena of band inversion, and present theoretical calculations and experimental angleresolved photoemission spectroscopy (ARPES) spectra, which support it in the form of electronic structure with a node at the Fermi energy. We also observe strongly temperature-dependent single-particle spectral weight and lineshape structure in ARPES, which suggest electronic correlations and coupling to collective modes.

\section{Results}

Fermi node state expected by band calculations. In support of this proposition, we first present $a b$ initio electronic structure calculations in the paramagnetic state. As detailed in the Supplementary Fig. 1, we show that the quadratic band touching systematically approaches the Fermi level with increasing A-site ionic radius, reaching it for $A=\operatorname{Pr}$. The corresponding band dispersion is shown in Fig. $2 \mathrm{~g}$ along the high-symmetry lines. The nodal Fermi point and quadratic band touching at $\Gamma$ is clearly visible at the Fermi energy. The bandwidth is narrower in energy than that of $\mathrm{HgTe}^{21}$ by one order of magnitude, reflecting the localized nature of the $5 d$ orbitals in $\operatorname{Pr}_{2} \mathrm{Ir}_{2} \mathrm{O}_{7}$. Theoretical uncertainty remains, however, as discussed in the Supplementary Note 1 (also see Supplementary Figs 1 and 2), so direct experimental evidence for the unique Fermi node state is strongly desired.

ARPES is a powerful technique to directly observe the electronic structure of matter ${ }^{22,23}$. One incident photon energy corresponds to one $k_{z}$ value in solid, thus the momentum space observed at a fixed photon energy is limited to a $k_{x}-k_{y}$ plane at a fixed $k_{z}$. To locate the $\Gamma$-point, therefore, sweeping the photon energy is required. Figure 2a,b shows the photos of the cleavage surface with the (111) plane measured by ARPES and the highquality single crystal we used, respectively. While the cleaved surface looks rough in the photo, the scanning electron microscope image of it (Fig. 2c,d) exhibits very flat parts in the multiple locations, which are large enough compared with the photon beam size $(\sim 150 \mu \mathrm{m}$, marked with a yellow circle in Fig. 2d). Several $k_{x}-k_{y}$ sheets perpendicular to $k_{(111)}$ measured at different photon energies are coloured in Fig. 2f. The band dispersions obtained by the $a b$ initio calculation for these sheets are plotted in Fig. $2 \mathrm{~h}-\mathrm{j}$. The experimentally obtained energy dispersion is expected to have a large gap when the $k_{(111)}$ location is far from the $\Gamma$. As $k_{(111)}$ is reduced, the gap decreases and eventually vanishes at $\Gamma$, where the two parabolic dispersions touch at $E_{\mathrm{F}}$ (Fig. 2j).

Observation of Fermi node in $\operatorname{Pr}_{2} \mathrm{Ir}_{2} \mathrm{O}_{7}$ by ARPES. To observe the Fermi node, we measured the ARPES spectra at various photon energies: $h v=7 \mathrm{eV}$ (a laser source) and $21.2 \mathrm{eV}$ (a He lamp) in the lab system, and $h v=8 \sim 18 \mathrm{eV}$ and $39 \sim 60 \mathrm{eV}$ from two different synchrotron facilities. The results for the 1st Brillouin zone (BZ) are shown in Fig. 3d, where the energy distribution curves (EDCs) along $k_{x}$ (defined in Fig. 3a) are plotted. The observed momentum cut shifts with photon energy along the $k_{(111)}$ axis within a momentum sheet crossing $\Gamma, \mathrm{L}$ and $\mathrm{K}$ points (coloured dashed lines in Fig. 3a). The spectra are symmetrized to remove the effect of the Fermi cutoff: the EDC is flipped about $E_{\mathrm{F}}$ and added to the original one. This method is widely accepted as a means of determining the presence of a gap

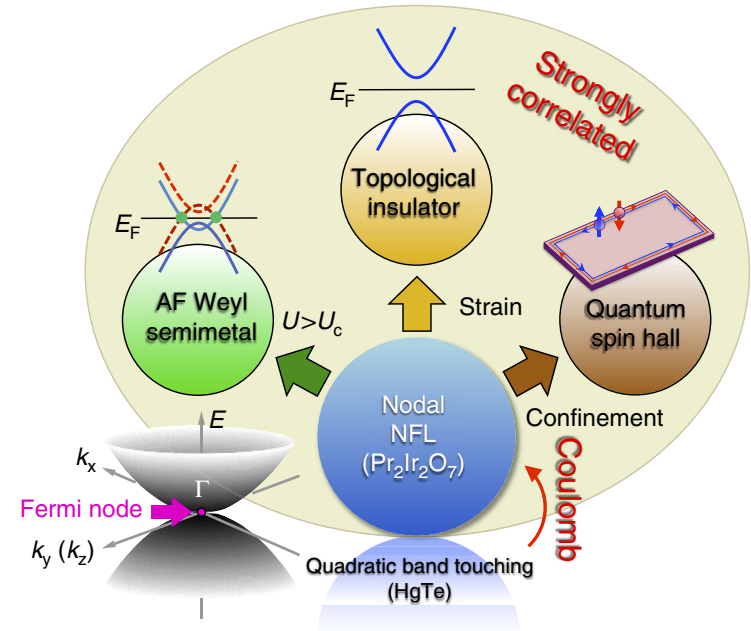

Figure 1 | Quadratic Fermi node state of $\mathrm{Pr}_{2} \mathrm{Ir}_{2} \mathrm{O}_{7}$ tunable into interacting topological phases. In the lower part of the diagram, the bottom half of the blue circle and its reflection form a caricature of the quadratically dispersion conduction and valence bands touching at the zone centre, while at the same time the darker-blue upper circle suggests how $\operatorname{Pr}_{2} \mid r_{2} \mathrm{O}_{7}$, with non-negligible Coulomb interactions, is a strongly correlated non-Fermi liquid analogue of $\mathrm{HgTe}$, shown as a pale-blue reflection. Arrows indicate the perturbations that convert the nodal non-Fermi liquid state to diverse topological phases: an antiferromagnetic Weyl semimetal should be produced in bulk materials by alloying or hydrostatic pressure, uniaxial strain yields a three-dimensional topological insulator and two-dimensional confinement produces a quantum spin Hall state. The outer circle reminds us that all the states produced in these ways retain strong correlations, and hence are excellent candidates for observing non-trivial surface phases different from those of band theory, as discussed in the text. 
a

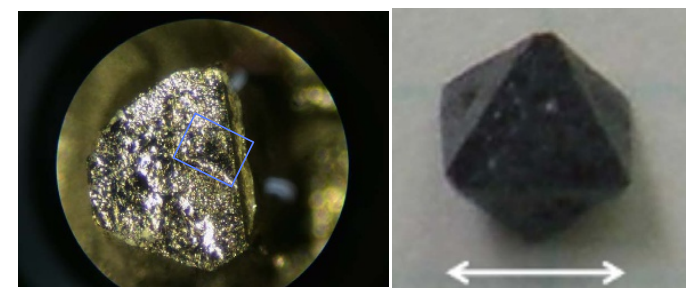

C

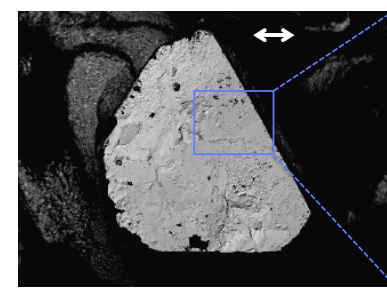

b

d

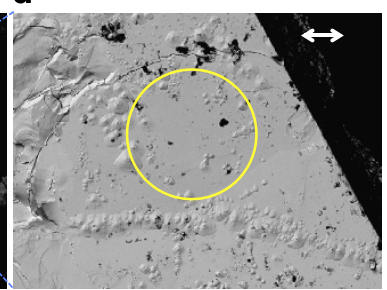

e

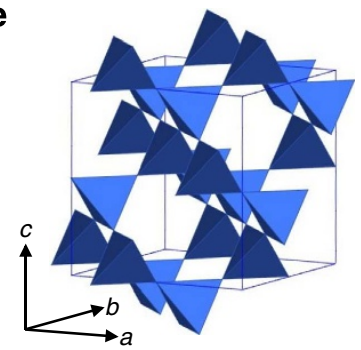

f

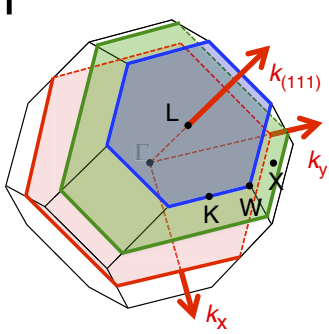

g

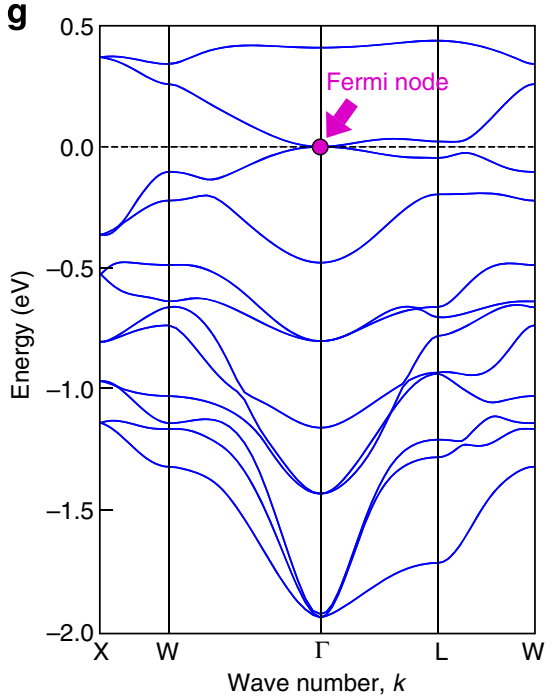

h
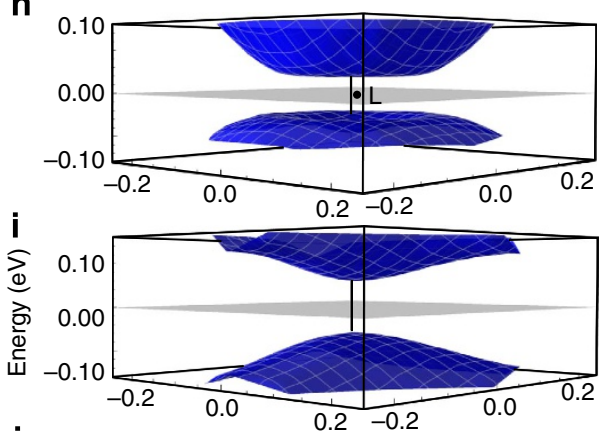

j

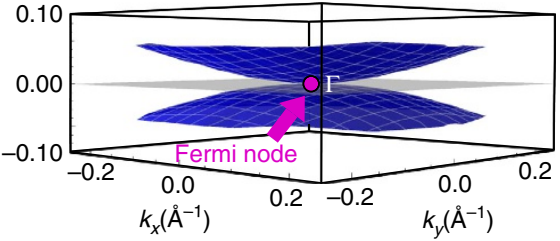

Figure 2 | Single-crystal and first-principles band calculation for $\mathbf{P r}_{\mathbf{2}} \mathbf{I r}_{\mathbf{2}} \mathbf{O}_{\mathbf{7}}$. (a,b) Photos of the cleaved (111) surface measured by ARPES and single crystal we used, respectively. The top surface with a triangle shape is the (111) plane. (c) The SEM image for the cleaved surface of a. (d) The same image as c, but magnified in a small region marked by a blue square in $\mathbf{a}$ and $\mathbf{c}$. The arrows in $\mathbf{b}, \mathbf{c}$ and $\mathbf{d}$ indicate dimensions of $1 \mathrm{~mm}, 200$ and $50 \mu \mathrm{m}$, respectively. (e) Crystal structure of $\mathrm{Pr}_{2} \mathrm{Ir}_{2} \mathrm{O}_{7}$ showing the pyrochlore lattice of $\mathrm{Ir}$ (or $\mathrm{Pr}$ ) atoms. (f) Brillouin zone. (g) Band dispersion along high-symmetry lines obtained by the first-principles calculation. Band dispersions in three $k_{x}-k_{y}$ planes (coloured in $\mathbf{f}$ ) perpendicular to the $k_{(111)}$ direction: crossing $L$ (h), crossing $\Gamma(\mathbf{j})$ and crossing between these two points (i). The Fermi node is indicated with a magenta circle and arrow in $\mathbf{g}$ and $\mathbf{j}$.

(or a gap node). Small but clear quasiparticle peaks are seen for all the spectra, which allows us to determine the energy dispersion, as marked by bars and dotted curves. At $h v=7 \mathrm{eV}$ (see Fig. 3d), a large gap of $\sim 20 \mathrm{meV}$ is opened at $k_{x}=0$ (green curve), and a parabolic dispersion is obtained. The most significant finding is that the parabolic dispersion moves towards the $E_{\mathrm{F}}$ with increasing incident photon energy (or increasing $k_{(111)}$ ), and it eventually touches $E_{\mathrm{F}}$ at $h v=10 \mathrm{eV}$. This behaviour is more clearly demonstrated in Fig. 3b,c, where the band dispersion determined from the peak energies of spectra at 7, 8, 9 and $10 \mathrm{eV}$ are plotted. As the photon energy is further increased, the dispersion moves away from $E_{\mathrm{F}}$ again, following the quadratic dispersion along $k_{(111)}$ as shown in Fig. 3e (also see Supplementary Figs 3 and 4; Supplementary Note 2). We have also examined the dispersion along a different momentum sheet crossing $\Gamma, \mathrm{L}$ and $\mathrm{W}$ points (see Supplementary Fig. 5 and Supplementary Note 2) for a different piece of sample. This time the photon energy was swept by a finer step $(\leq 0.5 \mathrm{eV})$, and the
Fermi node was again detected at $h v=10.5 \mathrm{eV}$. While it is not possible to eliminate a slight uncertainty in the exact photon energy, which yields $k_{(111)}=0$, owing to the broad shape of the spectral peak, our data show that the three-dimensional band structure of $\operatorname{Pr}_{2} \mathrm{Ir}_{2} \mathrm{O}_{7}$ has the theoretically predicted Fermi point at the momentum reached by $h v \sim 10 \mathrm{eV}$, which is thus assigned to be $\Gamma$. Other scans of different $k_{(111)}$ values up to the $\mathrm{L}$ point in the 1 st $\mathrm{BZ}$, which is reached at $h v=18 \mathrm{eV}$, revealed no other states touching or crossing $E_{\mathrm{F}}$ (see Supplementary Fig. 3). This absence of other bands crossing $E_{\mathrm{F}}$ is another consistency condition on the Fermi node model, which requires this situation by state counting and charge neutrality.

Here we validate our conclusion by making several further checks. First, we investigate another BZ to verify the required repetition of the Fermi nodal state along $k_{(111)}$. We used higher photon energies, corresponding to the 3rd BZ, and found the Fermi node at the expected $\Gamma$ point $(h v=52 \mathrm{eV})$ (see Supplementary Fig. 6). In the $k_{x}-k_{y}$ sheet at $h v=52 \mathrm{eV}$ (Fig. 4a,b), 
a
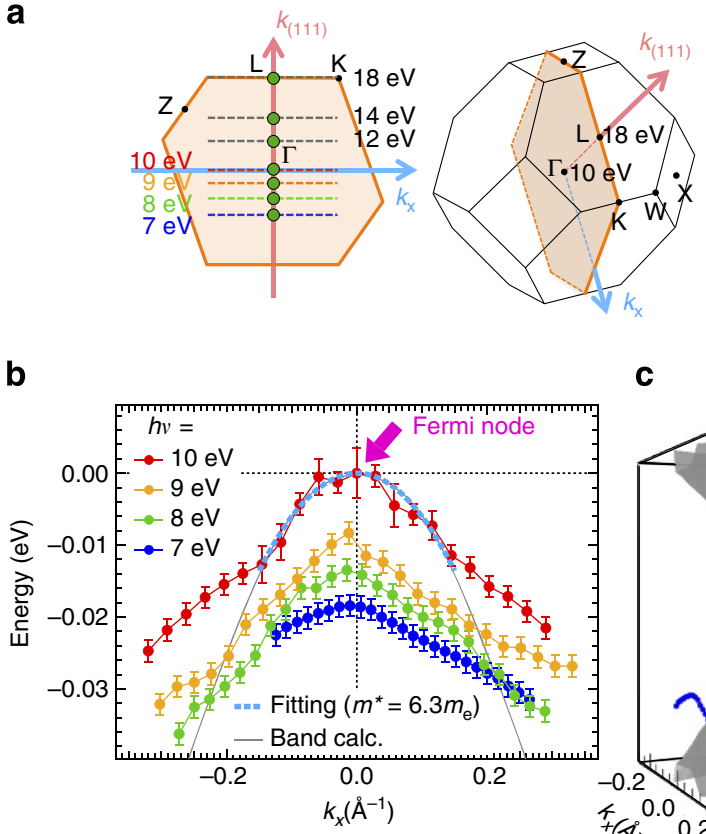

C $\quad k_{(111)}\left(\AA^{-1}\right)$
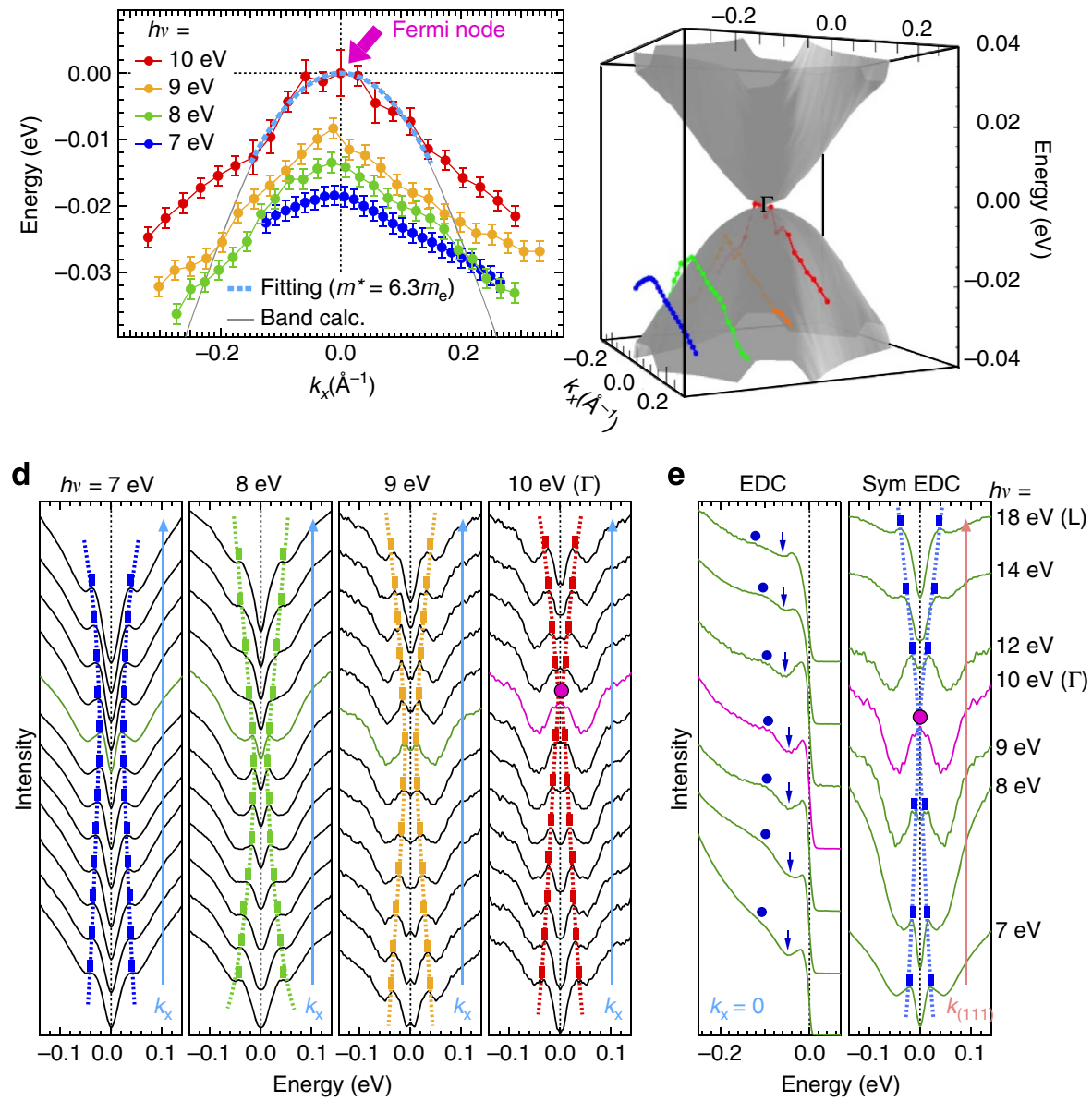

Figure 3 | ARPES spectra revealing a quadratic Fermi node in the 3D band of $\mathbf{P r}_{\mathbf{2}} \mathbf{I} \mathbf{r}_{\mathbf{2}} \mathbf{O}_{\mathbf{7}}$. (a) Brillouin zone, showing a momentum sheet along which ARPES data were measured. The momentum cuts at $h v=10$ and $18 \mathrm{eV}$ crosses the $\Gamma$ and $L$ points in the 1st Brillouin zone. (b) Energy dispersions along the $k_{x}$ direction measured at $h v=7,8,9$ and $10 \mathrm{eV}$. The corresponding momentum cuts are indicated in a by dashed coloured lines. The band dispersion obtained by the first-principles band calculation is superimposed (grey curve). The data close to $E_{\mathrm{F}}$ is fitted by a parabolic function, $\varepsilon(k) \propto k^{2}$ (light-blue dotted curve). The estimated effective mass at $\Gamma, m_{\text {eff }}=6.3 m_{0}\left(m_{0}\right.$ : free electron mass), is in agreement with the band calculation. (c) The calculated band dispersion in the $k_{x}-k_{(111)}$ sheet, painted with orange in $\mathbf{a}$. On it, the ARPES data in $\mathbf{b}$ are plotted. (d) The ARPES data (symmetrized energy distribution curves (EDCs)) along the $k_{x}$ direction measured at several photon energies. All the spectra shown were accumulated at $T=15 \mathrm{~K}$. The energy positions of spectral peaks are marked by bars and dashed curves. (e) EDCs and the corresponding symmetrized EDCs along the $\Gamma-\mathrm{L}$ direction, measured at $k$ points marked with green circles in $\mathbf{a}$. The energy positions of dip and hump, suggesting mode coupling, are marked by arrows and circles, respectively, on EDCs. Error bars in $\mathbf{b}$ represent uncertainty in estimating the spectral peak positions.

the ARPES intensities at $E_{\mathrm{F}}$ becomes strongest at the zone centre, and the band touching at $E_{\mathrm{F}}$ is confirmed in the dispersion maps along $k_{x}$ and $k_{y}$ (Fig. 3d) and the corresponding symmetrized EDCs (Fig. 4f, also see the raw EDCs in Supplementary Fig. 7). In contrast, these features are missing in the $k_{x}-k_{y}$ sheet across $\mathrm{L}$ point $(h v=39 \mathrm{eV})$ (see Fig. 4c), where a rather flat, gapped dispersion is observed (Fig. $4 \mathrm{e}, \mathrm{g}$ ).

Second, we demonstrate that our conclusion is insensitive to the different analytic schemes. This is significant especially because the symmetrization technique is relevant for the particle- hole symmetric state, which is unknown in $\operatorname{Pr}_{2} \mathrm{Ir}_{2} \mathrm{O}_{7}$. Accordingly, we have tested another widely used measure of dividing the ARPES spectra by the Fermi-Dirac function (FD). Figure $5 \mathrm{c}$ plots the EDCs along a momentum cut crossing $\Gamma$ (light-blue arrow in the inset of Fig. 5d) measured at various temperatures. Instead of symmetrization, the curves are divided by the energy-resolution convoluted Fermi function at the measurement temperatures to remove the effect of the Fermi cutoff. It is clearly seen that the quadratic dispersion touches $E_{\mathrm{F}}$, in agreement with the earlier analysis. In Fig. 5e, we compare the dispersions determined by the 

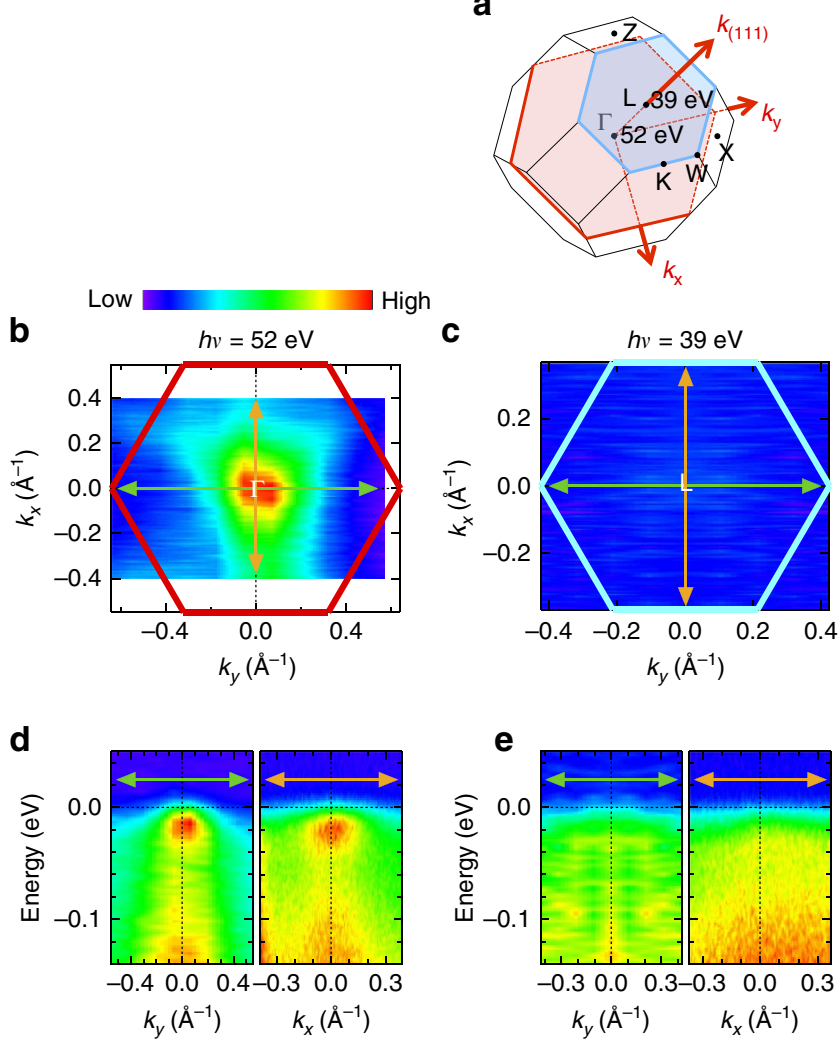

f

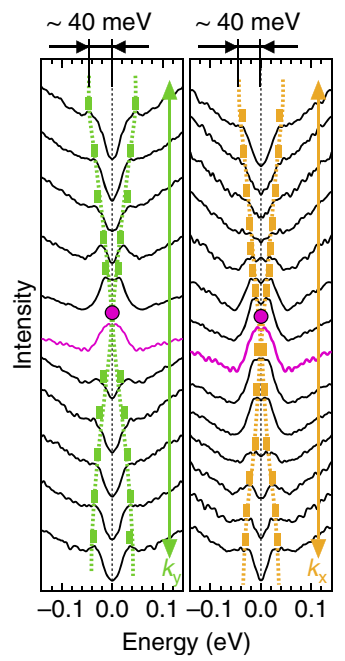

g

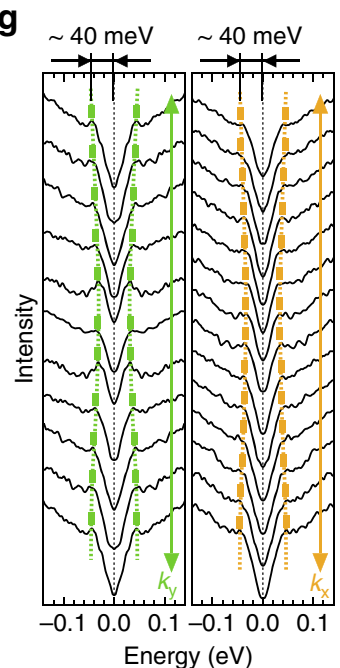

Figure 4 | ARPES intensity mapping at the Fermi level across $\boldsymbol{\Gamma}$ and $\mathbf{L}$ points. (a) Brillouin zone, showing momentum sheets along which ARPES data were measured. The momentum cuts at $h v=52$ and $39 \mathrm{eV}$ crosses the $\Gamma$ and $L$ points, respectively, in the 3rd Brillouin zone. $(\mathbf{b}, \mathbf{c})$ ARPES intensities at $E_{\mathrm{F}}$ in the $k_{x}-k_{y}$ sheet crossing $\Gamma$ and $\mathrm{L}$ measured at $h v=52$ and $39 \mathrm{eV}$, respectively. For the data at $h v=39 \mathrm{eV}$, only the positive side of $k_{y}$ were measured, and it is reflected to the negative side. (d) ARPES dispersion maps for $h v=52 \mathrm{eV}$, measured along $k_{y}$ and $k_{x}$ crossing $\Gamma$ (green and orange arrows in $\mathbf{b}$, respectively). (e) The same data as $\mathbf{d}$, but for $h v=39 \mathrm{eV}$, which cross the $\mathrm{L}$ point. $(\mathbf{f}, \mathbf{g})$ EDCs of $\mathbf{d}$ and $\mathbf{e}$, respectively, symmetrized about $E_{\mathrm{F}}$. The dimension arrows estimate the bandwidth of $\sim 40 \mathrm{meV}$ in the occupied side.

symmetrization and the FD-division methods, and confirm the consistency between the two (see Supplementary Fig. 8 and Supplementary Note 3 for more details).
Comparison between ARPES results and band calculations. In Fig. 3b, we compare our experimental results near $\Gamma$ with the $a b$ initio dispersion (grey curve), which has a purely quadratic shape of the electronic structure given by $\varepsilon(k) \propto k^{2}$. Close to $E_{\mathrm{F}}$, we find an agreement between the two, demonstrating that the quadratic curve (light-blue dotted line) fits well to our data with almost the same effective mass, $m_{\text {eff }}=6.3 m_{0}\left(m_{0}\right.$ is the mass of a free electron). On the other hand, for energy below $-0.012 \mathrm{eV}$, the measured dispersion deviates remarkably from the parabolic shape. This contrasts to the calculation, which matches with $\varepsilon(k) \propto k^{2}$ up to much higher binding energies. It is possible that the deviation is due to correlation effects beyond the band calculation. Intriguingly, the total bandwidth in the occupied side is estimated to be $\sim 40 \mathrm{meV}$ (arrows in Fig. $4 \mathrm{f}, \mathrm{g}$ ), which is actually much narrower than that $(>100 \mathrm{meV})$ of the band calculation (see Fig. 2g and Supplementary Fig. 9e,h). Band narrowing relative to the density functional theory is indeed a well-known characteristic of correlated electrons. If that is the case, however, the agreement of the effective mass around $\Gamma$ between the data and calculation would be a coincidence. This is understandable, considering that the band shape of $\mathrm{Pr}_{2} \mathrm{Ir}_{2} \mathrm{O}_{7}$ with comparable energy scales between the spin-orbit interaction and the electron correlations is sensitive to different calculation methods (see Supplementary Note 4; Supplementary Figs 1 and 9).

Another possible cause of the discrepancy between the data and calculations is the strong coupling of electrons to the bosonic modes, which also could significantly renormalize the band shape. Indeed, the peak-dip-hump shape, which is a characteristic signature of strong mode coupling, is seen in EDCs (Fig. 3e), being consistent with this scenario. One of candidates for the bosonic modes is the phonons, which are usually coupled to the correlated systems very strongly. Another candidate is suggested by the similarity of the slightly distorted band shape to measurements in graphene, where it was attributed to electronplasmon coupling ${ }^{24}$. Namely, the origin could be the same in the two cases: emission of collective modes through vertical interband transitions becomes possible above a threshold energy, modifying the spectrum.

Correlation-driven anomalous temperature evolution. The strongly correlated feature of $5 d$ electrons of $\operatorname{Pr}_{2} \mathrm{Ir}_{2} \mathrm{O}_{7}$ should be observed in the ARPES spectra. We find such a signature in the temperature evolution of the spectral shape. Figure $5 \mathrm{a}, \mathrm{b}$ plots Fermi-function-divided EDCs at $\Gamma(h v=10.5 \mathrm{eV})$ and off $\Gamma$ $\left(k_{(111)}=0.31 \AA^{-1}\right.$ reached at $\left.h v=21.2 \mathrm{eV}\right)$. The sharp peak clearly seen in the low-temperature spectra is strongly suppressed at elevated temperatures. The behaviour is clearly more marked than the thermal broadening effects observed in the other strongly collated systems such as the well-studied cuprates, which have the 'marginal' Fermi liquid state ${ }^{25,26}$. We note that the peak suppression in the present data is accelerated across $\sim 100 \mathrm{~K}$, differently from the typical thermal broadening with a gradual increase over a wider temperature range. We find that, associated with the suppression, a large portion of spectral weight above $E \simeq-0.1 \mathrm{eV}$ is transferred to higher binding energies in $\operatorname{Pr}_{2} \operatorname{Ir}_{2} \mathrm{O}_{7}$. A plausible origin for it is the polaronic effects, which could become crucial in the purely screened electronic systems, as also proposed for the other strongly correlated systems such as the perovskite iridates, manganites and lightly doped cuprates ${ }^{27-29}$. These features are compatible with the non-Fermi liquid behaviour predicted theoretically for the Fermi node phase in refs 11,30, and may also be related to recent observations of quantum critical behaviour in thermodynamic measurements of $\operatorname{Pr}_{2} \mathrm{Ir}_{2} \mathrm{O}_{7}$ (ref. 17). However, a 

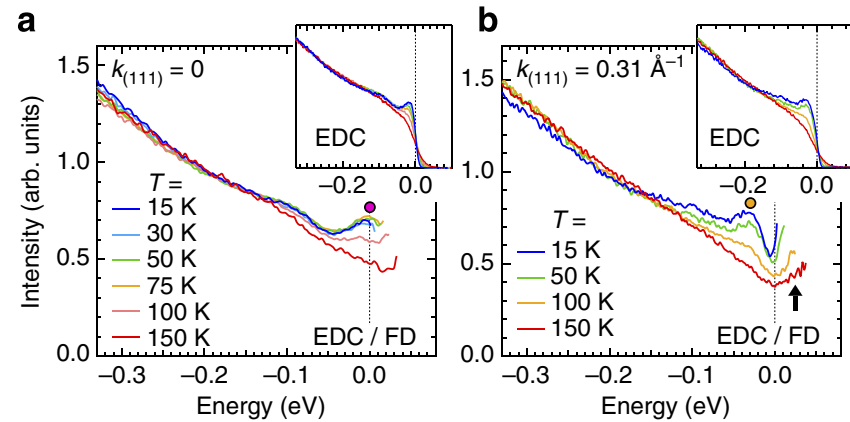

c

EDC/FD

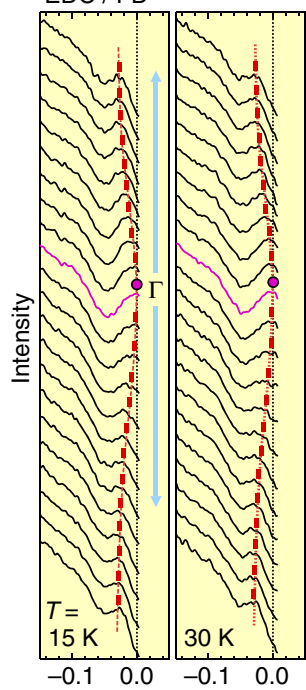

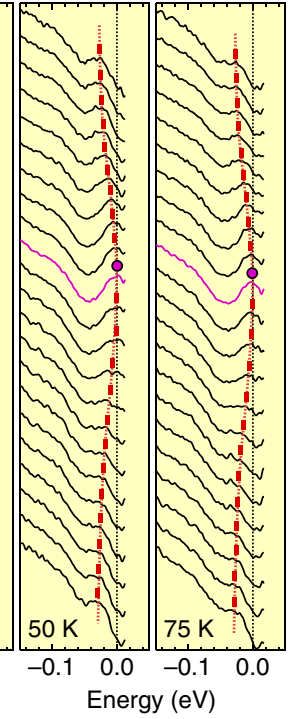

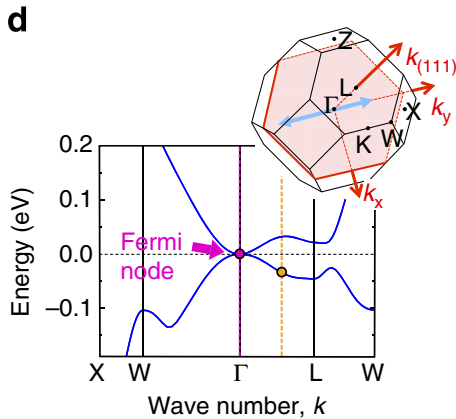

e
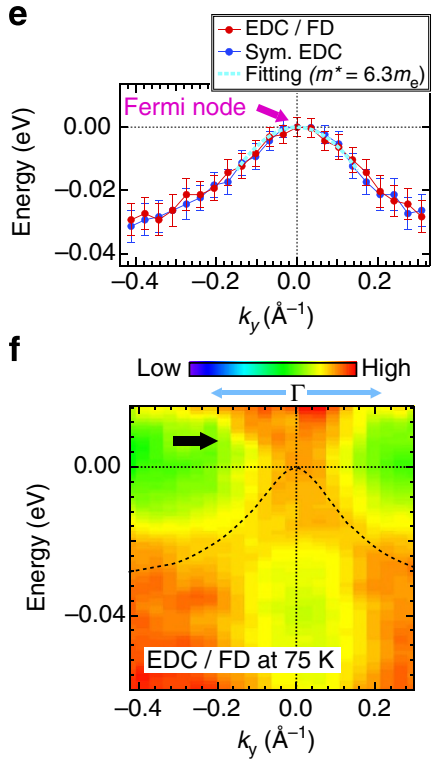

Figure 5 | Temperature evolution of ARPES spectra and evidence for the Fermi nodal state. (a,b) Temperature variation of FD-divided EDCs measured at $\Gamma(h v=10.5 \mathrm{eV})$ and off $\Gamma(h v=21.2 \mathrm{eV})$, respectively. In $\mathbf{d}$, the observed $k$ points for $\mathbf{a}$ and $\mathbf{b}$ are marked by purple and orange dashed lines, respectively. The unoccupied side is displayed up to the energy of $3 k_{B} T$. The spectral peak positions are marked by coloured circles. The original EDCs used for the analysis are plotted in the insets. (c) The FD-divided EDCs along a momentum cut indicated in d with a light-blue arrow measured at various temperatures. The spectral peak positions are indicated by red bars and dashed curves. The Fermi node is marked by a magenta circle. At $T=150 \mathrm{~K}$, the spectral peaks are strongly suppressed, and thus the dispersion cannot be determined. (d) Band dispersions obtained by the first-principles calculation, magnified in the region close to $E_{\mathrm{F} .}$ In the inset, the Brillouin zone of $\mathrm{Pr}_{2} \mathrm{Ir}_{2} \mathrm{O}_{7}$ is shown. (e) The energy dispersion along a momentum cut crossing $\Gamma$ (light-blue arrow in d), determined from the peak positions of the FD-divided spectra at $T=75 \mathrm{~K}$ in $\mathbf{c}$ (red circles). The same plots, but determined from the peak positions of symmetrized spectra, are superimposed (blue circles). The quadratic dispersion (light-blue dashed curve) nicely fits the data. (f) ARPES dispersion map crossing $\Gamma$ (light-blue arrow in $\mathbf{d}$ ) measured at $h v=10.5 \mathrm{eV}$. The original ARPES intensities are divided by the energy-resolution convoluted Fermi function at the measured temperature of $T=75 \mathrm{~K}$ to remove the cutoff effect near $E_{\mathrm{F}}$. The thick black arrow points to the remarkable spectral weight above $E_{\mathrm{F}}$ consistent with the existence of conduction touching band in the unoccupied side. Error bars in e represent uncertainty in estimating the spectral peak positions.

fuller identification of the non-Fermi liquid state and explication of its physics in $\operatorname{Pr}_{2} \mathrm{Ir}_{2} \mathrm{O}_{7}$ requires higher resolution data and more elaborate analysis, beyond the scope of this paper.

Quadratic band touching. In passing, we point out that broad spectral weight emerges beyond $E_{\mathrm{F}}$ as seen in the Fermi-functiondivided image for the 75- $\mathrm{K}$ data (arrow in Fig. $5 \mathrm{f}$ ). The related spectral intensity is obtained off $\Gamma$, showing an upturn behaviour beyond $E_{\mathrm{F}}$ (arrow in Fig. $5 \mathrm{~b}$ and Supplementary Figs 10 and 11, and see Supplementary Notes 5 and 6). While the strong suppression of quasiparticle peaks at elevated temperatures prevents us from a definitive determination of the conduction band dispersion, the observation of spectral weight above $E_{\mathrm{F}}$ is compatible with the predicted existence of a quadratic band touching on the unoccupied side (Fig. 5d).

\section{Discussion}

Prior measurements $6,16,31$ showing ferromagnetic spin-ice-type correlations among the Pr moments below $2 \mathrm{~K}$ may be explained due to unconventional ferromagnetic RKKY interactions arising from the point-like Fermi surface. The Fermi node also leads to strong sensitivity to small time-reversal breaking perturbations, producing Weyl points close to the Fermi energy ${ }^{12}$ and a gigantic anomalous Hall effect. This is also in accord with the experimental fact that $\operatorname{Pr}_{2} \mathrm{Ir}_{2} \mathrm{O}_{7}$ was the first material found to exhibit a large spontaneous Hall effect in a spin liquid state at zero field ${ }^{6,31}$.

Our results suggest that tuning of a unique quantum critical point between an antiferromagnetic Weyl semimetal and the nonFermi liquid nodal phase may be possible by alloying or hydrostatic pressure. Correlated topological phases and device applications with iridate films could be accessed by controlling the strain-induced breaking of the cubic crystal symmetry and 
size quantization (sub-band formation) in quantum well structures. We indeed verified theoretically the opening of a significant topological gap with uniaxial compression along the $\langle 111\rangle$ axis by first-principles calculations (see Supplementary Fig. 12 and Supplementary Note 7). This analysis, moreover, shows the presence of three two-dimensional surface Dirac cones in this TI state (see Supplementary Fig. 13; Supplementary Table 1; Supplementary Note 7). It will be exciting to investigate whether correlations, neglected in the density function theory, lead to spontaneous time-reversal breaking at surfaces ${ }^{32,33}$ with fractional excitations $s^{34}$, or surface topological order ${ }^{35-38}$, both of which have been predicted theoretically.

\section{Methods}

Samples and ARPES experiments. Single crystals of $\operatorname{Pr}_{2} \mathrm{Ir}_{2} \mathrm{O}_{7}$ with $1 \mathrm{~mm}^{3}$ size were grown using a flux method. The sample surface with the (111) plane was prepared by cleaving the single crystal in situ with a top post clued on the crystal.

The ARPES experiments were performed at BL7U of Ultraviolet Synchrotron Orbital Radiation (UVSOR) facility $(h v=8 \sim 18 \mathrm{eV})$ with a MBS A-1 electron analyzer $^{23}$, BL28A of Photon Factory, KEK $(h v=39 \sim 60 \mathrm{eV})$ with a Scienta SES2002 electron analyzer and in our laboratory using a system consisting of a Scienta R4000 electron analyzer equipped with a 6.994-eV laser (the 6th harmonic of $\mathrm{Nd}_{\mathrm{YVO}} \mathrm{YVO}_{4}$ quasi-continuous wave with a repetition rate of $\left.240 \mathrm{MHz}\right)^{22}$ and $\mathrm{He}$ discharge lamp $(\mathrm{HeI} \alpha, h v=21.2 \mathrm{eV})$. The overall energy resolution in the ARPES experiment was set to $\sim 2 \mathrm{meV}$ and $\sim 6 \mathrm{meV}$ in the lab system with a laser and $\mathrm{He}$ lamp, respectively, and $\sim 15 \mathrm{meV}$ for the synchrotron facility data. The sample orientation was determined with a Laue picture taken before the ARPES experiment.

The intrinsic $k_{z}$ broadening, $\delta k_{z}$, is inversely proportional to the photoelectron escape depth $\lambda\left(\delta k_{z}=1 / \lambda\right)$. We used low photon energies to find the nodal point in the 1st BZ, which enables the bulk sensitive measurements. For example, the photon energy corresponding to the $\Gamma$ point is around $10 \mathrm{eV}$, at which the $\lambda$-value is estimated to be $\sim 20 \AA$ according to the 'universal curve'. It translates into $\delta k_{z}$ with $\sim 5 \%$ of the BZ size, which is rather small and sufficient to validate our results. In the Supplementary Fig. 4, we demonstrate that the $\delta k_{z}$ (or $\delta k_{(111)}$ ) is small enough to resolve the $E$ versus $k_{(111)}$ relation with a $1-\mathrm{eV}$ step of photon energy. We also used higher photon energies of $\sim 50 \mathrm{eV}$ to investigate the $3 \mathrm{rd} \mathrm{BZ}$. In this case, the $\lambda$-value becomes small $(\sim 5 \AA)$, resulting in a relatively large $\delta k_{z}$ ( $\sim 20 \%$ of the BZ). Nevertheless, we note that the energy dispersion in $\operatorname{Pr}_{2} \mathrm{Ir}_{2} \mathrm{O}_{7}$ is very weak especially around the $\Gamma$ point (quadratic Fermi node), thus the effects of $k_{z}$ broadening on the quasiparticle peaks should not be critical in our study.

Band calculations. Calculations were carried out using both the standard generalized gradient approximation (GGA) and the Tran-Blaha modified Becke-Johnson (TB-mBJ) ${ }^{39}$ exchange potential as implemented in Wien2k, for a series of different A-site ions. An $\mathrm{RK}_{\max }$ parameter 7.0 was chosen and the wavefunctions were expanded in spherical harmonics up to $l_{\max }^{\mathrm{wf}}=10$ inside the atomic spheres and $l_{\max }^{\text {pot }}=4$ for non-muffin tins. Bulk $\mathrm{A}_{2} \mathrm{Ir}_{2} \mathrm{O}_{7}(\mathrm{~A}=$ rare-earth element) has a cubic crystal structure with the space group $\mathrm{Fd} \overline{3} \mathrm{~m}$. The experimental lattice parameters were used for $\mathrm{A}=\mathrm{Pr}, \mathrm{Nd}$, Eu and $\mathrm{Y}$ in both GGA and TB-mBJ paramagnetic calculations, and spin-orbit coupling was applied to both the heavy rare-earth element and the Ir electrons. The paramagnetic GGA and TB-mBJ calculations put the $4 \mathrm{f}$ states of the rare-earth element at the Fermi energy; to avoid this, since the 4 f electrons are highly localized, their potential is shifted by a constant.

The TB-mBJ method is believed to produce improved results for small band gap systems ${ }^{40}$, and has been widely used in studies of topological insulators ${ }^{41}$. Both methods (GGA and TB-mBJ) yield almost indistinguishable results away from the Fermi energy, with a slight decrease of bandwidth in the TB-mBJ calculation, and small differences near $E_{F}$, as shown in Supplementary Fig. 1. We observed the symmetry-required nodal band touching at $\Gamma$ for all calculations, but the Fermi energy is shifted from the nodal point by an amount that decreases with increasing $\mathrm{A}(\mathrm{A}=\mathrm{Y}, \mathrm{Eu}, \mathrm{Nd}$ and $\mathrm{Pr})$ site ionic radius in both methods ${ }^{42}$. This occurs because of accidental crossing of states near the $\mathrm{L}$ point, where the valence band rises and approaches the Fermi energy, especially in the smaller rare earths. In general, the GGA calculation underestimates the effects of correlations, which tend to narrow the bands and to push occupied states deeper below the Fermi energy. Hence, our expectation is that the accidental crossing near $\mathrm{L}$, which is responsible for the Fermi level shift in GGA, will be suppressed by correlations. The TB-mBJ method may be regarded as a crude way to do this. Indeed, in the presumed more accurate TB-mBJ method, all the paramagnetic band structures for the $\mathrm{A}_{2} \mathrm{Ir}_{2} \mathrm{O}_{7}$ series show smaller shifts of the Fermi level compared with their GGA counterparts. In the case of $\mathrm{A}=\mathrm{Pr}$, we find the shift vanishes and the nodal point occurs precisely at the Fermi energy. While there is a universal trend of a smaller shift of the Fermi energy as rare-earth ionic radius increases for the rare-earth elements we have tested, the small differences between GGA and TB-mBJ suggest that some theoretical uncertainties remain. We note, however, that we expect methods that include correlations more accurately, such as combined local density approximation and dynamical mean field theory (LDA + DMFT), are likely to further suppress the states near the Fermi energy at L even beyond TB-mBJ, favouring placing the Fermi level precisely at the node.

\section{References}

1. Hasan, M. Z. \& Kane, C. L. Colloquium: topological insulators. Rev. Mod. Phys 82, 3045-3067 (2010).

2. Fu, L., Kane, C. L. \& Mele, E. J. Topological insulators in three dimensions Phys. Rev. Lett. 98, 106803 (2007).

3. Moore, J. E. \& Balents, L. Topological invariants of time-reversal-invariant band structures. Phys. Rev. B 75, 121306 (2007).

4. Ando, Y. Topological insulator materials. J. Phys. Soc. Jpn 82, 102001 (2013).

5. Kim, B. et al. Novel $\mathrm{J}_{\text {eff }}=1 / 2$ Mott state induced by relativistic spin-orbit coupling in $\mathrm{Sr}_{2} \mathrm{IrO}_{4}$. Phys. Rev. Lett. 101, 076402 (2008).

6. Machida, Y., Nakatsuji, S., Onoda, S., Tayama, T. \& Sakakibara, T. Timereversal symmetry breaking and spontaneous Hall effect without magnetic dipole order. Nature 463, 210-213 (2009).

7. Witczak-Krempa, W. \& Kim, Y. B. Topological and magnetic phases of interacting electrons in the pyrochlore iridates. Phys. Rev. B 85, 045124 (2012)

8. Guo, H.-M. \& Franz, M. Three-dimensional Topological Insulators on the Pyrochlore Lattice. Phys. Rev. Lett. 103, 206805 (2009).

9. Pesin, D. \& Balents, L. Mott physics and band topology in materials with strong spin-orbit interaction. Nat. Phys. 6, 376-381 (2010).

10. Witczak-Krempa, W., Chen, G., Kim, Y.-B. \& Balents, L. Correlated quantum phenomena in the strong spin-orbit regime. Annu. Rev. Condens. Matter Phys. 5, 57-82 (2014).

11. Moon, E.-G., Xu, C., Kim, Y. B. \& Balents, L. Non-Fermi-liquid and topological states with strong spin-orbit coupling. Phys. Rev. Lett. 111, 206401 (2013).

12. Wan, X., Turner, A. M., Vishwanath, A. \& Savrasov, S. Y. Topological semimetal and Fermi-arc surface states in the electronic structure of pyrochlore iridates. Phys. Rev. B 83, 205101 (2011).

13. Yanagishima, D. \& Maeno, Y. Metal-nonmetal changeover in pyrochlore iridates. J. Phys. Soc. Jpn 70, 2880-2883 (2001).

14. Matsuhira, K. et al. Metal-insulator transition in pyrochlore iridates $\operatorname{Ln}_{2} \mathrm{Ir}_{2} \mathrm{O}_{7}$ (Ln = Nd, Sm, and Eu). J. Phys. Soc. Jpn 76, 043706 (2007).

15. Nakatsuji, S. et al. Metallic spin-liquid behavior of the geometrically frustrated Kondo lattice $\operatorname{Pr}_{2} \mathrm{Ir}_{2} \mathrm{O}_{7}$. Phys. Rev. Lett. 96, 087204 (2006).

16. Machida, Y. et al. Unconventional anomalous Hall effect enhanced by a noncoplanar spin texture in the frustrated Kondo lattice $\operatorname{Pr}_{2} \mathrm{Ir}_{2} \mathrm{O}_{7}$. Phys. Rev. Lett. 98, 057203 (2007).

17. Tokiwa, Y., Ishikawa, J. J., Nakatsuji, S. \& Gegenwart, P. Quantum criticality in a metallic spin liquid. Nat. Mater. 13, 356-359 (2014).

18. Sun, K., Yao, H., Fradkin, E. \& Kivelson, S. A. Topological insulators and nematic phases from spontaneous symmetry breaking in 2D Fermi systems with a quadratic band crossing. Phys. Rev. Lett. 103, 046811 (2009).

19. Bernevig, B. A., Hughes, T. L. \& Zhang, S.-C. Quantum spin Hall effect and topological phase transition in HgTe quantum wells. Science 314, 1757-1761 (2006).

20. Brüne, C. et al. Quantum Hall effect from the topological surface states of strained bulk HgTe. Phys. Rev. Lett. 106, 126803 (2011).

21. Zaheer, S. et al. Spin texture on the Fermi surface of tensile-strained HgTe. Phys. Rev. B 87, 045202 (2013).

22. Kiss, T. et al. A versatile system for ultrahigh resolution, low temperature, and polarization dependent Laser-angle-resolved photoemission spectroscopy. Rev. Sci. Instrum. 79, 023106-023106 (2008).

23. Kimura, S.-I. et al. SAMRAI: a novel variably polarized angle-resolved photoemission beamline in the VUV region at UVSOR-II. Rev. Sci. Instrum. 81, 053104-053104 (2010).

24. Bostwick, A., Ohta, T., Seyller, T., Horn, K. \& Rotenberg, E. Quasiparticle dynamics in graphene. Nat. Phys. 3, 36-40 (2006).

25. Varma, C. M., Littlewood, P. B., Schmitt-Rink, S., Abrahams, E. \& Ruckenstein, A. E. Phenomenology of the normal state of $\mathrm{Cu}-\mathrm{O}$ high-temperature superconductors. Phys. Rev. Lett. 63, 1996-1999 (1989).

26. Kondo, T., Takeuchi, T., Tsuda, S. \& Shin, S. Electrical resistivity and scattering processes in $(\mathrm{Bi}, \mathrm{Pb})_{2}(\mathrm{Sr}, \mathrm{La})_{2} \mathrm{CuO}_{6}+\delta$ studied by angle-resolved photoemission spectroscopy. Phys. Rev. B 74, 224511 (2006).

27. King, P. D. C. et al. Spectroscopic indications of polaronic behavior of the strong spin-orbit insulator $\mathrm{Sr}_{3} \mathrm{Ir}_{2} \mathrm{O}_{7}$. Phys. Rev. B 87, 241106 (2013).

28. Dessau, D. S. et al. $k$-Dependent electronic structure, a large 'ghost' Fermi Surface, and a pseudogap in a layered magnetoresistive oxide. Phys. Rev. Lett. 81, 192-195 (1998).

29. Shen, K. M. et al. Missing quasiparticles and the chemical potential puzzle in the doping evolution of the cuprate superconductors. Phys. Rev. Lett. 93, 267002 (2004)

30. Savary, L., Moon, E.-G. \& Balents, L. New type of quantum criticality in the pyrochlore iridates. Phys. Rev. X 4, 041027 (2014). 
31. Balicas, L., Nakatsuji, S., Machida, Y. \& Onoda, S. Anisotropic hysteretic Hall effect and magnetic control of chiral domains in the chiral spin states of $\mathrm{Pr}_{2} \mathrm{Ir}_{2} \mathrm{O}_{7}$. Phys. Rev. Lett. 106, 217204 (2011).

32. Baum, Y. \& Stern, A. Magnetic instability on the surface of topological insulators. Phys. Rev. B 85, 121105 (2012).

33. $\mathrm{Xu}, \mathrm{C}$. Time-reversal symmetry breaking at the edge states of a threedimensional topological band insulator. Phys. Rev. B 81, 020411 (2010).

34. Qi, X.-L., Hughes, T. L. \& Zhang, S.-C. Fractional charge and quantized current in the quantum spin Hall state. Nat. Phys. 4, 273-276 (2008).

35. Wang, C., Potter, A. C. \& Senthil, T. Gapped symmetry preserving surface state for the electron topological insulator. Phys. Rev. B 88, 115137 (2013).

36. Metlitski, M. A., Kane, C. L. \& Fisher, M. P. A. Symmetry-respecting topologically ordered surface phase of three-dimensional electron topological insulators. Phys. Rev. B 92, 125111 (2015).

37. Chen, X., Fidkowski, L. \& Vishwanath, A. Symmetry enforced non-Abelian topological order at the surface of a topological insulator. Phys. Rev. B 89, 165132 (2014).

38. Bonderson, P., Nayak, C. \& Qi, X.-L. A time-reversal invariant topological phase at the surface of a 3D topological insulator. J. Stat. Mech. 2013, 09016 (2013).

39. Tran, F. \& Blaha, P. Accurate band gaps of semiconductors and insulators with a semilocal exchange-correlation potential. Phys. Rev. Lett. 102, 226401 (2009).

40. Koller, D., Tran, F. \& Blaha, P. Merits and limits of the modified Becke-Johnson exchange potential. Phys. Rev. B 83, 195134 (2011).

41. Feng, W., Xiao, D., Zhang, Y. \& Yao, Y. Half-Heusler topological insulators: a first-principles study with the Tran-Blaha modified Becke-Johnson density functional. Phys. Rev. B 82, 235121 (2010).

42. Ishii, F. et al. First-principles study on cubic pyrochlore iridates $\mathrm{Y}_{2} \mathrm{Ir}_{2} \mathrm{O}_{7}$ and $\mathrm{Pr}_{2} \mathrm{Ir}_{2} \mathrm{O}_{7}$. J. Phys. Soc. Jpn 84, 073703 (2015).

\section{Acknowledgements}

This work was supported by JSPS KAKENHI (Nos 24740218, 25220707 and 25707030), by the Photon and Quantum Basic Research Coordinated Development Program from MEXT, by PRESTO, Japan Science and Technology Agency, Grants-in-Aid for Scientific Research (No. 25707030), by Grants-in-Aids for Scientific Research on Innovative Areas (15H05882 and15H05883), and Program for Advancing Strategic International Networks

to Accelerate the Circulation of Talented Researchers (No. R2604) from the Japanese Society for the Promotion of Science. L.B. and R.C. were supported by DOE grant DE-FG02-08ER46524, and E.-G.M. was supported by the MRSEC Program of the NSF under Award No. DMR 1121053. We thank D. Hamane for technical assistance in the SEM measurement. The use of the facilities (the Materials Design and Characterization Laboratory and the Electronic Microscope Section) at the Institute for Solid State Physics, The University of Tokyo, is gratefully acknowledged.

\section{Author contributions}

T.K., S.N., L.B. and S.S. designed the experiment. T.K., M.N., H.K., Y.N., T.Y., Y.O., W.M., Y.I., R.Y. and H.Y. carried out the ARPES experiment, and M.M., S.K., N.I., K.O and H.K. assisted with measurements at the synchrotron radiation facilities. T.K. and M.N. performed the data analysis. J.J.I. and S.N. grew the high-quality single crystals. R.C., E.-G.M. and L.B. carried out the calculations. T.K., R.C. and L.B. wrote the manuscript. All authors discussed the results and commented on the manuscript.

\section{Additional information}

Supplementary Information accompanies this paper at http://www.nature.com/ naturecommunications

Competing financial interests: The authors declare no competing financial interests.

Reprints and permission information is available online at http://npg.nature.com/ reprintsandpermissions/

How to cite this article: Kondo, T. et al. Quadratic Fermi node in a 3D strongly correlated semimetal. Nat. Commun. 6:10042 doi: 10.1038/ncomms10042 (2015).

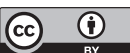

International License. The images or other third party material in this article are included in the article's Creative Commons license, unless indicated otherwise in the credit line; if the material is not included under the Creative Commons license, users will need to obtain permission from the license holder to reproduce the material. To view a copy of this license, visit http://creativecommons.org/licenses/by/4.0/ 